\title{
Christian Rohr: Historische Hilfswissenschaften. Eine Einfüh- rung. Wien [u. a.]: Böhlau Verlag 2015.
}

Lange Zeit war eine leicht lesbare, fundierte Einführung in die Historischen Hilfswissenschaften ein Desideratum. Der österreichische Historiker Christian Rohr hat nun diese Lücke mit der Einführung „Historische Hilfswissenschaften" geschlossen.

Bevor Rohr 2010 als Professor für Umwelt- und Klimageschichte ans Historische Institut der Universität Bern berufen wurde, war er an der Universität Salzburg tätig und hielt dort die Lehrveranstaltung „Klassische Arbeitstechniken in der Geschichtswissenschaft". Auf dieser Lehrtätigkeit basiert das vorliegende Werk.

Das Handbuch gliedert die historischen Hilfswissenschaften in Quellenkunde, Diplomatik, Paläographie, Archiv- und Aktenkunde, Historische Geographie und Regionalforschung, „Kleine Hilfswissenschaften“ wie Numismatik, Metrologie, Heraldik und Genealogie.

Ein kurzes abschließendes Kapitel befasst sich mit den Hilfswissenschaften und dem WWW. Schon alleine die Tatsache, dass die Fachbegriffe der Kapitelüberschriften in Klammern erklärt werden zeigt, dass sich das Handbuch (auch) an Studienanfängerinnen und -anfänger richtet. Am Ende jedes (Teil-) Kapitels findet sich ein entsprechendes Literaturverzeichnis, das wesentlich zur Übersichtlichkeit und zum komfortablen Arbeiten beiträgt.

Rohr stellt die Geschichtsquellen nicht nur in einen gesamthistorischen Zusammenhang, sondern behandelt auch die Geschichte der einzelnen Hilfswissenschaften. Damit ist das vorliegende Handbuch auch ein Beitrag zur europäischen Wissenschafts- bzw. Geistesgeschichte. Von Beginn an versucht das Handbuch, Studierende zur Äußeren und Inneren Quellenkritik zu ermutigen, indem es sowohl formale Kriterien des Quellenmaterials ausführlich behandelt als auch nach der Motivation ihrer Entstehung fragt.

Der Schwerpunkt der Publikation liegt bei den „klassischen“ Hilfswissenschaften, nämlich der Diplomatik und der Paläographie, die rund zwei 
Drittel des Textes einnehmen. Besonders interessiert dabei den Mediävisten Rohr naturgemäß das Mittelalter. Sehr ausführlich werden verschiedene Formen und Funktionen von Urkunden vorgestellt. Großer Wert wird dabei sowohl auf den formalen Aufbau als auch die innere Quellenkritik gelegt. Die in der Verlagsaussendung aufgeworfene Frage: „Warum wurden im Mittelalter Urkunden gefälscht?" beantwortet Rohr anhand zweier Beispiele, die österreichischen respektive Salzburger Studierenden geläufig sein sollten, nämlich das Privilegium maius und die Pilgrim'sche Schenkung. Zwei ergänzende Unterkapitel zur Diplomatik befassen sich mit der Sphragistik und der Chronologie.

Für den bibliothekarischen Bereich ist besonders das Kapitel über die Chronologie von Bedeutung, da auch die Datierung gedruckter historischer Quellen mitunter Probleme aufwerfen kann. Rohr gibt einen Überblick über Kalendersysteme in verschiedenen Kulturen von der griechischen Antike bis zur Französischen Revolution. Im Zentrum steht der Klassiker der Zeitrechnung, nämlich Hermann Grotefends „Taschenbuch der Zeitrechnung", von dem bereits eine überarbeitete Online-Version existiert.

Das Kapitel zur Paläographie bringt nach einem Überblick über die Geschichte der Beschreibstoffe und der Schreibwerkzeuge, die in Antike und Mittelalter verwendet wurden, eine kurze Terminologie und ein Verzeichnis der in der Spätantike und im Mittelalter verwendeten Abkürzungen. Sehr ausführlich widmet sich das Handbuch den „wichtigsten Buch- und Urkundenschriften des Mittelalters“, dem Buchschmuck und der äußeren Form wie Lagen und Einbänden. Darauf baut die Handschriftenbeschreibung auf, die sehr prägnant und übersichtlich erklärt wird.

Knapp wird die Schriftenkunde der Neuzeit behandelt, das Kapitel schließt mit der Abschaffung der „deutschen Schrift“ durch die Nationalsozialisten. Zahlreiche Schriftbeispiele von der Spätantike bis ins 19. Jahrhundert illustrieren den Text, Transkriptionen ermöglichen den Studierenden, die eigenen paläographischen Kenntnisse zu überprüfen.

Sehr praxisorientiert wird das Thema „Historisches Arbeiten im Archiv“, etwa wenn Rohr seinen Leserinnen und Lesern auf ihren Archivbesuch eine Art Handlungsleitfaden mitgibt, dessen erster Satz lautet: „Gehen Sie mit dem Archivpersonal immer höflich und nicht fordernd um. Man ist Ihnen sicher gerne behilflich, wird Ihnen aber nicht genau die Textstelle, nach der Sie suchen, herausfinden können, " ein Ratschlag, der auch gut auf Bibliotheken anwendbar ist.

Ein wirkliches Defizit des an sich sehr lesenswerten und gut lesbaren Handbuchs ist die sehr knapp gehaltene Auseinandersetzung mit Internetressourcen wie etwa im Kapitel Genealogie (Familien- und Abstammungs- 
kunde), in dem durchaus verlässliche Onlinequellen unerwähnt bleiben, wie überhaupt das Kapitel „Historische Hilfswissenschaften und das WWW“ selbst mit gerade einmal zwei Seiten äußerst kurz ausfällt. Rohr gibt kaum Links an, da sich diese „,häufig ändern oder nicht mehr aktiv sind." Er empfiehlt stattdessen den durchaus pragmatischen Weg, relevante Seiten über Suchmaschinen aufzurufen. Dem ist entgegenzuhalten, dass große Archive, Bibliotheken oder andere wissenschaftliche Einrichtungen, von denen anzunehmen ist, dass ihre Web-Adressen stabil sind, Portale betreiben, die durchaus Werkzeuge für die Historischen Hilfswissenschaften zur Verfügung stellen, wie beispielsweise die E-Learning-Angebote des Instituts für Geschichte der Universität Wien oder die Linksammlungen für Genealogen des Österreichischen Staatsarchivs.

Auch wenn diese Einführung den Anspruch hat, historische Hilfswissenschaften von der Römerzeit bis zur Gegenwart abzuhandeln, liegt der Fokus eindeutig auf dem Mittelalter. Da keine Vorkenntnisse vorausgesetzt werden und die Materie fundiert und kenntnisreich vermittelt wird, bietet das vorliegende Buch auch Studienanfängerinnen und -anfängern einen niederschwelligen Einstieg in die Arbeit mit Geschichtsquellen, die ja das eigentliche Metier der Historiker ist. Darüber hinaus ist es aufgrund der klaren Gliederung auch als Nachschlagewerk bestens geeignet und wird gewiss einen fixen Platz in den Regalen von Bibliotheken mit historischen Beständen finden.

Isabella Wasner-Peter, Wien

Dieses Werk ist lizenziert unter einer

Creative-Commons-Lizenz Namensnennung 4.0 International 